\title{
DESENVOLVIMENTO INICIAL A CAMPO DE MUDAS DE CAFÉ ARÁBICA CATUAÍ VERMELHO PROVENIENTES DE DIFERENES RECIPIENTES E SUBSTRATOS
}

\author{
Matheus Wandermurem da Silva ${ }^{1}$ \\ Jean Karlos Barros Galote ${ }^{2}$ \\ Juliana Elias de Oliveira ${ }^{3}$ \\ Sandy Queiroz Espinoso ${ }^{4}$ \\ Diego Souza Tosta ${ }^{5}$ \\ Richardson Sales Rocha ${ }^{6}$ \\ Otacílio José Passos Rangel ${ }^{7}$ \\ Wallace Luís de Lima ${ }^{8}$
}

Resumo: Com o objetivo de avaliar os efeitos de recipientes e substratos orgânicos no desenvolvimento inicial de cafeeiros (Coffea arabica L.). O experimento foi realizado no Setor de Agroecologia do Ifes Campus de Alegre, em delineamento experimental inteiramente casualizado, em esquema fatorial duplo (2x4), sendo o primeiro fator o tipo de recipientes (sacolinha de polietileno - $650 \mathrm{~cm}^{3}$; tubetinho $-120 \mathrm{~cm}^{3}$ ), o segundo fator aos tipos de substratos orgânicos (Plantmax; Composto A (Leguminosa); Composto B (Orgânico); Composto C (Vermicomposto)). Foram produzidas 48 mudas seminais de café arábica Catuaí vermelho (IAC 44), cada tratamento com 6 repetições, e parcela constituída de 1 planta. As melhores respostas para as variáveis de desenvolvimento avaliadas, foram obtidas para as mudas oriundas de sacolinhas $\left(650 \mathrm{~cm}^{3}\right)$.

Palavras-chave: Cafeicultura agroecológica; Compostagem; Manejo cultural.

\footnotetext{
1 Curso Superior de Tecnologia em Cafeicultura/Instituto Federal do Espírito Santo - Ifes Campus de Alegre, Brasil. E-mail: matheus_wandermurem@hotmail.com.

2 Pós-Graduação em Agroecologia/Instituto Federal do Espírito Santo - Ifes Campus de Alegre, Brasil. E-mail: jean-karlos10@hotmail.com.

3 Engenharia Agronômica/Universidade Federal do Espírito Santo Engenharia em Agronomia, Brasil. E-mail: juliana.elias.o@hotmail.com.

${ }_{4}^{4}$ Curso Superior de Tecnologia em Cafeicultura/Instituto Federal do Espírito Santo - Ifes Campus de Alegre, Brasil. E-mail: sandyespinoso@gmail.com.

5 Curso Superior de Tecnologia em Cafeicultura/Instituto Federal do Espírito Santo - Ifes Campus de Alegre, Brasil. E-mail: diego.s.tosta@gmail.com.

${ }^{6}$ Curso Superior de Tecnologia em Cafeicultura/Instituto Federal do Espírito Santo - Ifes Campus de Alegre, Brasil. E-mail: richardsonsales2016@gmail.com.

${ }^{7}$ Professor/Instituto Federal do Espírito Santo - Ifes Campus de Alegre, Brasil. E-mail: otaciliorangel@gmail.com.

${ }^{8}$ Professor/Instituto Federal do Espírito Santo - Ifes Campus de Alegre, Brasil. E-mail: limawl@yahoo.com.br.
} 